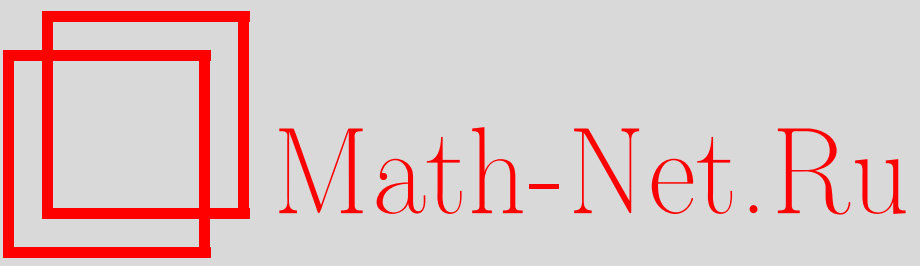

В. Р. Халилов, Фермион в кулоновском и магнитном полях в $2+1$ измерениях, ТМФ, 1999, том 119, номер 1, 105-118

DOI: https://doi.org/10.4213/tmf731

Использование Общероссийского математического портала Math-Net.Ru подразумевает, что вы прочитали и согласны с пользовательским соглашением

http://www . mathnet.ru/rus/agreement

Параметры загрузки:

IP : 54.224 .60 .19

26 апреля 2023 г., 16:13:45 
ТЕОРЕТИЧЕСКАЯ

И МАТЕМАТИЧЕСКАЯ

ФИЗИКА

Том 119, № 1

апрель, 1999

(C) 1999 г.

\section{ФЕРМИОН В КУЛОНОВСКОМ И МАГНИТНОМ ПОЛЯХ В $2+1$ ИЗМЕРЕНИЯХ}

Построены квазиклассические решения и спектр энергий уравнения Дирака в сильном кулоновском и слабом постоянном однородном магнитном полях в $2+1$ измерениях применительно к проблеме двумерного водородоподобного атома, помещенного во внешнее магнитное поле. Найдены некоторые “точные" решения уравнений Дирака и Паули, которые описывают "бесспиновые" фермионы в сильных кулоновском и однородном магнитном полях в $2+1$ измерениях.

\section{1. ВВЕДЕНИЕ}

Системы нерелятивистских электронов во внешнем электромагнитном поле, движение которых ограничено в плоскости, привлекают большой интерес в связи с возможностью применения описывающих их теоретико-полевых моделей для изучения таких замечательных квантовых макроскопических явлений, как квантовый эффект Холла и высокотемпературная сверхпроводимость $[1,2]$. Ряд эффектов в физике конденсированных сред указывает на существование $(2+1)$-мерных систем со спектром энергий, определяемым гамильтонианом уравнения Дирака [3]. В частности, вырожденный плоский полупроводник с низкоэнергетической динамикой электронов, как предполагают, можно хорошо описать с помошью $(2+1)$-мерной релятивистской теории Дирака [4]. В связи с этим изучение физических эффектов, протекающих в $(2+1)$-мерных системах заряженных частиц в присутствии сильных внешних полей, актуально в настоящее время с точки зрения возможных практических приложений.

Здесь мы найдем квазиклассические и некоторые точные решения уравнений Паули и Дирака во внешних кулоновском и магнитном полях в $2+1$ измерениях. Физической реализацией изучаемых моделей могут служить системы электронов, движение которых ограничено в плоскости. Известно [5], что точных решений этих уравнений в терминах специальных функций не существует, однако, как показано в работах [6, 7], в нерелятивистском случае можно найти аналитические решения, которые представимы в виде произведения некоторой весовой функции и конечного полинома. Такие решения

* Московский государственный университет, Москва, Россия. E-mail:khalilov@thc.phys.msu.su 
называют точными. Точные решения уравнения Дирака и эффект рождения пар заряженных фермионов из вакуума сильным кулоновским полем протяженного источника в $2+1$ измерениях обсуждались недавно в статье [8].

В данной статье в основном используется система единиц, в которой $c=\hbar=1$, однако мы восстанавливаем размерности физических величин в ряде мест, где это необходимо.

\section{2. УРАВНЕНИЕ ДИРАКА ВО ВНЕШНЕМ ЭЛЕКТРОМАГНИТНОМ ПОЛЕ В $2+1$ ИЗМЕРЕНИЯХ}

Поскольку [3] в $2+1$ измерениях алгебра матриц Дирака может быть представлена в терминах матриц Паули как $\gamma^{0}=\sigma^{3}, \gamma^{k}=i \sigma^{k}$, уравнение Дирака для электрона массы $m$ и заряда $e=-e_{0}, e_{0}>0$, взаимодействие которого с внешним полем удовлетворяет принципу "минимальности", имеет вид

$$
\left(i \partial_{t}-H_{\mathrm{D}}\right) \Psi=0
$$

где

$$
H_{\mathrm{D}}=\alpha \mathbf{P}+\beta m+e A^{0} \equiv \sigma^{1} P_{2}-\sigma^{2} P_{1}+\sigma^{3} m+e A^{0}
$$

- гамильтониан, $P_{\mu}=i \partial_{\mu}-e A_{\mu}$ - оператор обобщенного импульса электрона, $A_{\mu}$ - вектор-потенциал внешнего электромагнитного поля, а полный момент импульса электрона имеет только одну компоненту $J_{z}=L_{z}+S_{z}$, где $L_{z}=-i \partial / \partial \varphi$ и $S_{z}=\sigma^{3} / 2$.

Для того чтобы включить спин электрона в одночастичную теорию дираковского поля в $3+1$ измерениях, используют только четные части соответствующих спиновых операторов, которые не смешивают состояния с различными знаками энергии. Спиновые операторы должны иметь необходимые ковариантные свойства и быть интегралами движения. Четная часть некоторого оператора $\bar{A}$ определяется формулой

$$
\bar{A}=\frac{H_{\mathrm{D}} A+A H_{\mathrm{D}}}{2 E} .
$$

Если внешнее электромагнитное поле однородно, предпочтение отдается так называемому оператору тензора поляризации [9]

$$
T_{d}^{\mu \nu}=\frac{i}{2} \Psi^{\dagger} \gamma^{0}\left(\gamma^{\mu} \gamma^{\nu}-\gamma^{\nu} \gamma^{\mu}\right) \Psi
$$

В $2+1$ измерениях для используемого представления $\gamma$-матриц выбранньй спиновьй оператор имеет только следующие матричные компоненты:

$$
T^{01}=-\sigma^{1}, \quad T^{02}=-\sigma^{2}, \quad T^{12}=I,
$$

где $I$ - двухрядная диагональная единичная матрица.

Рассмотрим для определенности движение электрона во внешнем однородном постоянном магнитном поле напряженности $B$, вектор-потенциал которого выберем в калибровке Ландау $A^{0}=A^{1}=0, A^{2}=B x$. Спиновый оператор $T^{12}$ в таком поле является 
интегралом движения. Полагая $\Psi(t, \mathbf{x})=\exp (-i \epsilon E t+i p y) \psi(x)$, где $\epsilon= \pm 1$ - знак, а $E>0$ - абсолютное значение энергии, и подставляя $\Psi$ в уравнение $(1)$, нетрудно найти спектр энергии

$$
E_{n}=\epsilon \sqrt{m^{2}+2 n|e B|}
$$

и две компоненты $\psi(x)$ в виде

$$
\psi(x)=\left(\begin{array}{c}
c_{1} u_{n-1}(\eta) \\
c_{2} u_{n}(\eta)
\end{array}\right)
$$

Здесь функции

$$
u_{n}(\eta)=\frac{\left(e_{0} B\right)^{1 / 4}}{\left(2^{n} n ! \pi^{1 / 2}\right)^{1 / 2}} \exp \left(-\frac{\eta^{2}}{2}\right) H_{n}(\eta)
$$

выражаются через полиномы Эрмита $H_{n}(\eta)$ аргумента $\eta=\left(e_{0} B\right)^{1 / 2} x+p /\left(e_{0} B\right)^{1 / 2}$, целое число $n(n=0,1,2, \ldots)$ указывает номер уровня Ландау, а коэффициенты $c_{i}$ удовлетворяют условию нормировки $\left|c_{1}\right|^{2}+\left|c_{2}\right|^{2}=1$.

Подставляя в формулу (3) величины $H_{\mathrm{D}}$ и $E$ из соотношений $(2)$ и $(6)$, получим $\bar{T}^{12}=$ $H_{\mathrm{D}} / E \equiv \mu_{B}$, а для спинового квантового числа фермиона находим

$$
\frac{\mu_{B}}{2} \Psi=\frac{1}{2} \epsilon \Psi
$$

Отсюда видно, что спиновое квантовое число фермиона при фиксированных $e$ и $B$ может иметь только одно значение. Это означает, что, в отличие от случая трех пространственных измерений, в $2+1$ измерениях нет вырождения уровней Ландау по спину, т.е. фактически $(2+1)$-мерные электроны ведут себя подобно бесспиновым фермионам.

В нерелятивистском приближении волновая функция $(2+1)$-мерных фермионов имеет лиш одну компоненту, если уравнение Паули выводится из уравнения (1); $\Psi$ можно представить в виде $[10,11]$

$$
\Psi=\exp \left(-\frac{i m c^{2} t}{\hbar}\right) \psi
$$

Здесь мы восстановили фундаментальные костанты: $c$ - скорость света и $\hbar$-постоянную Планка. В результате в первом приближении по $1 / c$ получим уравнение

$$
\left(i \hbar \frac{\partial}{\partial t}-e A_{0}\right) \psi=\left(\frac{P_{1}^{2}+P_{2}^{2}}{2 m}-\frac{e \hbar}{2 m c} F_{12}\right) \psi,
$$

где $P_{k}=-i \hbar \partial / \partial x^{k}-e A_{k} / c$.

Ниже мы найдем некоторые решения уравнений (1) и (10) во внешнем кулоновском поле, описываемом вектор-потенциалом

$$
A^{0}(r)=-\frac{Z e_{0}}{r}, \quad A^{x}=A^{y}=0,
$$

и однородном постоянном магнитном поле напряженности $B$, вектор-потенциал которого выберем в симметричной калибровке как

$$
A^{0}=0, \quad A^{x}=-\frac{B y}{2}, \quad A^{y}=\frac{B x}{2} .
$$

Вначале имеет смысл кратко проанализировать особенности движения релятивистского электрона во внешнем кулоновском поле точечного источника [8]. 


\section{3. ДВИЖЕНИЕ ЭЛЕКТРОНА В ПОЛЕ ТОЧЕЧНОГО ЯДРА}

Решение уравнения Дирака (1) в полях (11), (12) ишем в виде

$$
\Psi(t, \mathbf{x})=\frac{1}{\sqrt{2 \pi}} \exp (-i \epsilon E t+i l \varphi) \psi(r, \varphi)
$$

где $\epsilon= \pm 1$ - знак, $E>0$ - абсолютное значение энергии, $l$ - целое число и

$$
\psi(r, \varphi)=\left(\begin{array}{c}
f(r) \\
g(r) e^{i \varphi}
\end{array}\right)
$$

Заметим, что функция $\psi(r, \varphi)$ является собственной функцией оператора полного момента $J_{z}$ с собственным значением $l+1 / 2$.

Подставляя выражения (13) и (14) в уравнение (1) и учитывая равенства

$$
P_{x} \pm i P_{y}=-i e^{ \pm i \varphi}\left(\frac{\partial}{\partial r} \pm\left(\frac{i}{r} \frac{\partial}{\partial \varphi}+\frac{e B r}{2}\right)\right)
$$

получим следующую систему уравнений:

$$
\begin{array}{r}
\frac{d f}{d r}-\left(\frac{l}{r}-\frac{e B r}{2}\right) f+\left(\epsilon E+m+\frac{Z \alpha}{r}\right) g=0, \\
\frac{d g}{d r}+\left(\frac{1+l}{r}-\frac{e B r}{2}\right) g-\left(\epsilon E-m+\frac{Z \alpha}{r}\right) f=0,
\end{array}
$$

где $\alpha \equiv e^{2}=1 / 137$ - постоянная тонкой структуры.

Точные решения и спектр энергий уравнения Дирака в кулоновском поле точечного ядра в $2+1$ измерениях можно найти следующим образом [8]. Положим в уравнениях $(16) B=0$, а функции $f(r)$ и $g(r)$ представим в виде

$$
\begin{aligned}
& f=\sqrt{m+E} e^{-\rho / 2} \rho^{\gamma-1}\left(Q_{1}+Q_{2}\right), \\
& g=\sqrt{m-E} e^{-\rho / 2} \rho^{\gamma-1}\left(Q_{1}-Q_{2}\right),
\end{aligned}
$$

где введены обозначения

$$
\rho=2 \lambda r, \quad \lambda=\sqrt{m^{2}-E^{2}}, \quad \gamma=\frac{1}{2}+\sqrt{\left(l+\frac{1}{2}\right)^{2}-(Z \alpha)^{2}}, \quad \alpha \equiv e^{2},
$$

а $\gamma$ определяет асимптотическое поведение волновых функций при малых $r$.

Решение уравнений (16) при $B=0$, конечное при $\rho=0$, выражается через вырожденную гипергеометрическую функцию $F(a, b ; z)$ :

$$
\begin{aligned}
& Q_{1}=A F\left(\gamma-\frac{1}{2}-\left(\frac{Z \alpha E}{\lambda}\right), 2 \gamma ; \rho\right), \\
& Q_{2}=D F\left(\gamma+\frac{1}{2}-\left(\frac{Z \alpha E}{\lambda}\right), 2 \gamma ; \rho\right),
\end{aligned}
$$


причем связь между постоянными $A$ и $D$ задается соотношением

$$
D=\frac{\gamma-\frac{1}{2}-\frac{Z \alpha E}{\lambda}}{l+\frac{1}{2}+\frac{Z \alpha m}{\lambda}} A
$$

Спектр энергий определяется из уравнения

$$
\gamma-\frac{1}{2}-\frac{Z \alpha E}{\lambda}=-n_{r}
$$

Нетрудно показать, что допустимы следующие значения квантового числа $n_{r}: n_{r}=$ $0,1,2, \ldots$ при $l \geqslant 0$ и $n_{r}=1,2,3, \ldots$ при $l<0$. Следовательно, дискретный спектр энергий электрона в поле (11) имеет вид

$$
E=m\left[1+\frac{(Z \alpha)^{2}}{\left(n_{r}+\sqrt{\left(l+\frac{1}{2}\right)^{2}-(Z \alpha)^{2}}\right)^{2}}\right]^{-1 / 2}
$$

Этот спектр энергий похож на спектр энергий бесспиновой заряженной частицы в кулоновском поле [11] в трех пространственных измерениях, что и можно было ожидать, т.к. в $(2+1)$-мерной квантовой теории электрон фактически ведет себя подобно бесспиновому фермиону. Однако в отличие от энергии истинного бозона энергия бесспинового электрона на низшем уровне $\left(l=n_{r}=0\right)$ есть

$$
E_{0}=m \sqrt{1-(2 Z \alpha)^{2}}
$$

и обрашается в нуль при $Z \alpha=1 / 2$.

Для нахождения энергетического спектра электрона в кулоновском поле в этом случае необходимо поставить некоторое граничное условие при $r=0$, т.е. следует рассматривать потенциал, обрезанный на некотором расстоянии $R$ [12-15], что эквивалентно учету конечных размеров ядра, создающего кулоновский потенциал.

\section{4. ФЕРМИОН В КУЛОНОВСКОМ И СЛАБОМ МАГНИТНОМ ПОЛЯХ}

Исключая из системы (16) функцию $g(r)$, получим

$$
\begin{aligned}
\frac{d^{2} f(r)}{d r^{2}} & +\frac{1}{r} \frac{d f(r)}{d r}+\left(E^{2}-m^{2}-|e B|(l+1)+\frac{2 \epsilon E Z \alpha}{r}+\frac{(Z \alpha)^{2}-l^{2}}{r^{2}}-\frac{(e B r)^{2}}{4}\right) f(r)+ \\
& +\frac{Z \alpha}{r^{2}}\left(\epsilon E+m+\frac{Z \alpha}{r}\right)^{-1}\left(\frac{d f(r)}{d r}-\left(\frac{|e B| r^{2}+2 l}{2 r} f(r)\right)\right)=0 .
\end{aligned}
$$

Отметим, что последний член этого уравнения пропорционален $\hbar$. Этот член мал при достаточно больших $r$, однако он влияет на асимптотику решений при очень малых 
$r \sim \hbar / m c$. Учитывая это, в последнем члене уравнения (24) сохраним только главные члены. Затем, вводя функцию $F(r)=r f(r)$, преобразуем уравнение $(24)$ к виду

$$
\frac{d^{2} F(r)}{d r^{2}}+\left(K+\frac{2 E Z \alpha}{r}+\frac{(Z \alpha)^{2}-l(l+1)}{r^{2}}-\frac{(e B r)^{2}}{4}\right) F(r)=0
$$

где

$$
K=E^{2}-m^{2}-|e B|\left(l+\frac{3}{2}\right)
$$

Подчеркнем, что уравнение (25) практически точно описьвает движение электрона в кулоновском поле точечного источника и слабом магнитном поле лишь при малых $r$.

Легко видеть, что как при положительных, так и при отрицательных $K$ функция $F(r) \sim r^{\gamma}$ при малых $r$, где $\gamma$ определяется формулой (18). Рассмотрим случай отрицательных $K$ и предел относительно слабого магнитного поля. При этом последний член в уравнении (25) можно опустить, и тогда уравнение (25) имеет точное решение, стремящееся к нулю при $r \rightarrow \infty$, которое можно выразить в виде функции Уитеккера

$$
F(r) \sim W_{\beta, \frac{\nu}{2}}(2 \lambda r)
$$

где

$$
\beta=\frac{E Z \alpha}{\lambda}, \quad \nu=2 \sqrt{\left(l+\frac{1}{2}\right)^{2}-(Z \alpha)^{2}}, \quad \lambda=\sqrt{m^{2}-E^{2}+|e B|\left(l+\frac{3}{2}\right)} .
$$

Функцию (27) можно записать в виде произведения вырожденной гипергеометрической функции $F(a, b ; z)$ и асимптотических решений, соответствуюших малым и большим $r$, следующим образом:

$$
W_{\beta, \mu}(z)=\frac{\Gamma(-2 \mu)}{\Gamma\left(\frac{1}{2}-\mu-\beta\right)} M_{\beta, \mu}+\frac{\Gamma(2 \mu)}{\Gamma\left(\frac{1}{2}+\mu-\beta\right)} M_{\beta,-\mu}
$$

где

$$
M_{\beta, \pm \mu}=z^{ \pm \mu+1 / 2} e^{-z / 2} F\left( \pm \mu-\beta+\frac{1}{2}, \pm 2 \mu+1 ; z\right)
$$

Конечное при $r=0$ для произвольных $l$ решение, очевидно, определяется первым членом правой части уравнения (29). Спектр энергий формируется на больших расстояниях, поэтому гипергеометрическая функция $F(a, b ; z)$ должна сводиться к полиному при больших $z$, что произойдет, если параметр $a$ равен целому отрицательному числу или нулю. Дискретный спектр энергий электрона, если определять его с помощью решений уравнения (25), имеет вид

$$
E=\left[m+\frac{|e B|}{2 m}\left(l+\frac{3}{2}\right)\right]\left[1+\frac{(Z \alpha)^{2}}{\left(n_{r}+\frac{1}{2}+\sqrt{\left(l+\frac{1}{2}\right)^{2}-(Z \alpha)^{2}}\right)^{2}}\right]^{-1 / 2}
$$


В пределе слабого магнитного поля решения и дискретные уровни энергии с $E<m$ можно также найти прямо для системы (16) в квазиклассическом приближении. Для этого, заменив $l$ на $l+1 / 2$ в первом из уравнений системы (16), что необходимо для получения правильного значения фазы квазиклассической волновой функции при больших $r$, будем искать решения этой системы в стандартном виде

$$
f(r)=A(r) \exp (i S(r)), \quad g(r)=B(r) \exp (i S(r)) .
$$

Здесь $A(r)$ и $B(r)$ - медленно меняюшиеся функшии. Подставляя (32) в (16), получим обыкновенное дифференциальное уравнение для определения функции $S(r)$ в нулевом приближении по $\hbar$ в виде

$$
\left(\frac{d S}{d r}\right)^{2} \equiv Q^{2}=E^{2}-m^{2}-e B\left(l+\frac{1}{2}\right)+\frac{2 E Z \alpha}{r}+\frac{(Z \alpha)^{2}-\left(l+\frac{1}{2}\right)^{2}}{r^{2}}-\frac{(e B r)^{2}}{4}
$$

Дискретные уровни энергии с $E<m$ определяются интегралом

$$
\int_{r_{\min }}^{r_{\max }} Q d r=\pi\left(-\sqrt{\left(l+\frac{1}{2}\right)^{2}-(Z \alpha)^{2}}+\frac{E Z \alpha}{\sqrt{\left|m^{2}+e B\left(l+\frac{1}{2}\right)-E^{2}\right|}}\right),
$$

где $r_{\max }>r_{\min }-$ классические точки поворота. При вычислении интеграла и корней подкоренного выражения член, пропорциональный $(e B r)^{2}$, в величине $Q$ не учитывался. Если потребовать, чтобы квазиклассическое выражение для спектра сводилось к формуле (22) при $B=0$, мы должны приравнять правую часть формулы (34) величине $\pi n_{r}$. Тогда в пределе слабого магнитного поля для дискретных уровней энергии с $E<m$ и для $l \neq 0$ получим

$$
E=\left[m+\frac{|e B|}{2 m}\left(l+\frac{1}{2}\right)\right]\left[1+\frac{(Z \alpha)^{2}}{\left(n_{r}+\sqrt{\left(l+\frac{1}{2}\right)^{2}-(Z \alpha)^{2}}\right)^{2}}\right]^{-1 / 2} .
$$

Как и следовало ожидать, эта формула для спектра энергий отличается от полученной выше (в соответствии с уравнением (25)) формулы (31).

В нерелятивистском приближении выражение для спектра энергий принимает вид

$$
E_{\mathrm{non}}=\frac{|e B|}{2 m}\left(l+\frac{1}{2}\right)-\frac{(Z \alpha)^{2}}{2\left(n_{r}+\left|l+\frac{1}{2}\right|\right)^{2}} .
$$

Для нахождения энергетических уровней при $l=0$ нужно найти точное решение уравнения (25) вблизи точки $r=0$ и сшить его с квазиклассическим решением при больших $r$. Для больших квантовых чисел $E \approx m$ точное решение уравнения $(25) \mathrm{c} l=0$, без учета члена $(e B r)^{2}$, в приближении $Z \alpha \ll 1$, вблизи $r=0$ имеет вид

$$
F=\sqrt{r} J_{1}(\sqrt{8 m Z \alpha r})
$$


Учитывая, что при $l=0$ имеем $r_{\min }=0$, и сшивая решение $(37)$ при $r \gg 1 / m Z \alpha$ с квазиклассическим решением уравнения (25), получим следуюшее правило квантования:

$$
\int_{0}^{r_{\max }} \sqrt{E^{2}-m^{2}-\frac{3|e B|}{2}+\frac{2 E Z \alpha}{r}+\left(\frac{Z \alpha}{r}\right)^{2}}=\pi n .
$$

Квазиклассическое движение электрона в магнитном и кулоновском полях (для определенности мы имеем в виду поле притяжения) можно охарактеризовать с помощью величин $l_{B}=\sqrt{2 \hbar c /|e B|}$ ("магнитная длина") и $a_{\mathrm{B}}=m \hbar^{2} / Z e^{2}$ (радиус Бора водородоподобного атома заряда $Z e)$. В приближении слабого магнитного поля $l_{B} \gg a_{\mathrm{B}}$ или, что эквивалентно, $B \ll B_{\mathrm{cr}}(Z \alpha)^{2} \equiv m^{2} c^{3} / e_{0} \hbar(Z \alpha)^{2} \equiv B_{0}$, т.е. движение электрона определяется кулоновским полем, а спектр энергий формируется на расстояниях порядка $a_{\mathrm{B}}$ и является, по сушеству, “релятивистским "спектром водородоподобного атома, возмушенного слабым магнитным полем. Это приводит к зеемановскому расщеплению спектра, которое линейно по напряженности магнитного поля и пропорционально “магнитному квантовому числу" $l+1 / 2$.

\section{5. ДВИЖЕНИЕ ЭЛЕКТРОНА В СИЛЬНЫХ КУЛОНОВСКОМ И МАГНИТНОМ ПОЛЯХ}

Рассмотрим теперь некоторые точные решения релятивистского уравнения (25) в сильных кулоновском и магнитном полях, когда $B \sim B_{0}$. Так как теперь в уравнении $(25)$ нельзя пренебрегать членом $(e B r)^{2} / 2$, то при больших $r$ асимптотическое решение $F(r) \sim \exp \left(-a r^{2} / 2\right)$, где $a=|e B| / 2$. Представим решения уравнения (25) с $\epsilon=1$ в виде произведения полинома и асимптотических решений (при малых и больших $r$ ) в виде

$$
F(r)=r^{\gamma} \exp \left(-\frac{a r^{2}}{2}\right) \sum_{n=0} c_{n} r^{n} .
$$

Подставляя ряд (39) в уравнение (25), получим

$$
\sum_{n=0} c_{n} r^{n+\gamma} \exp \left(-\frac{a r^{2}}{2}\right)\left[\left(n^{2}+n(2 \gamma-1)\right) r^{-2}+P+2 E Z \alpha r^{-1}\right]=0
$$

где

$$
P=E^{2}-m^{2}-2 a(n+l+2+\gamma)
$$

Из формулы (40) находим

$$
\begin{gathered}
c_{0} \neq 0, \\
2 E Z \alpha c_{0}+2 \gamma c_{1}=0
\end{gathered}
$$

и $($ для $n \geqslant 2)$

$$
\left[n^{2}+n(2 \gamma-1)\right] c_{n}+2 E Z \alpha c_{n-1}+\left[E^{2}-m^{2}-2 a(n+l+\gamma)\right] c_{n-2}=0
$$


Мы получили трехчленное рекуррентное соотношение. Заметим, что, записав его в форме

$$
\left[n^{2}+n(2 \gamma-1)\right] \frac{c_{n}}{c_{n-1}}+2 E Z \alpha+\left[E^{2}-m^{2}-2 a(n+l+\gamma)\right] \frac{c_{n-2}}{c_{n-1}}=0
$$

можно получить цепную дробь, позволяющую в принципе связать параметры уравнения, для которых можно найти решение. Однако анализ получаемого с помошью цепной дроби соотношения между параметрами сложен, поэтому для небольших $n$ проше получить решения прямо из соотношений (42), (43) и (44).

Из рекуррентного соотношения (44) следует, что решение становится полиномом $(n-1)$-й степени, если потребовать выполнения условий $c_{n}=c_{n+1}=0$. Остальные коэффициенты $c_{n+k}$ для любого положительного целого $k$ исчезают автоматически. Из (44) можно также получить соотношение между параметрами, при котором решения становятся полиномиальными,

$$
E^{2}-m^{2}=2 a(n+1+l+\gamma)
$$

Простейшие решения (для $n=2,3)$ нетрудно получить в аналитическом виде. Так, из соотношений (43), (44) и (46) найдем энергию

$$
\begin{aligned}
& E_{2}=m\left(1-\frac{4(Z \alpha)^{2}(3+l+\gamma)}{2 \gamma}\right)^{-1 / 2}, \\
& E_{3}=m\left(1-\frac{2(Z \alpha)^{2}(4+l+\gamma)}{1+6 \gamma}\right)^{-1 / 2},
\end{aligned}
$$

а также соотношения между величиной напряженности магнитного поля и другими параметрами, при которых решения в виде полиномов первой и второй степеней, соответственно, сушествуют:

$$
\begin{aligned}
& e B=\frac{\left(2 E_{2} Z \alpha\right)^{2}}{2 \gamma} \text { при } n=2, \\
& e B=\frac{\left(E_{3} Z \alpha\right)^{2}}{1+6 \gamma} \text { при } n=3 .
\end{aligned}
$$

Для простейших решений с $n=2,3$ значения параметров $E^{2}, B$, при которых соответствующие коэффициенты полиномов $c_{n}, c_{n+1}, \ldots, c_{n+k}$ обрашаются в нуль, единственны. Для $n \geqslant 4$ число различных значений этих параметров, а значит, и число различных решений равно $n / 2[7,16]$. Тем не менее для каждого $n$ имеется решение, являюшееся ортогональным полиномом степени $n-1$.

Эти решения представляют особый интерес, т.к. согласно известным теоремам [17] каждый ортогональный полином $n$-го порядка ортогонален (с определенной весовой функцией) всем полиномам меньшего порядка, а нули каждого элемента семейства ортогональных полиномов действительны и различны. В частности, ортогональный полином $n$-го порядка имеет $n$ нулей. Заметим, что именно для ортогональных полиномов 
значения параметров $E^{2}, B$ минимальны. Нелишне подчеркнуть, что только решения в виде полиномов гарантируют нормированность собственных функций.

В нерелятивистском приближении из соотношений $(47),(49)$ и $(48),(50)$ найдем соответствуюшие значения энергии

$$
\begin{aligned}
& E_{2}=\frac{2 m\left(Z e^{2}\right)^{2}}{\hbar^{2}(1+|2 l+1|)}\left(3+l+\frac{1}{2}+\left|l+\frac{1}{2}\right|\right), \\
& E_{3}=\frac{m\left(Z e^{2}\right)^{2}}{\hbar^{2}(4+|6 l+3|)}\left(4+l+\frac{1}{2}+\left|l+\frac{1}{2}\right|\right),
\end{aligned}
$$

а также соотношения между значениями напряженности магнитного поля и параметрами, характеризуюшими кулоновское взаимодействие и проекцию орбитального момента электрона, при которых решения в виде полиномов сушествуют,

$$
\begin{array}{llll}
\hbar \omega & =4 m c^{2} \frac{(Z \alpha)^{2}}{1+|2 l+1|} \quad \text { при } & n=2, \\
\hbar \omega=2 m c^{2} \frac{(Z \alpha)^{2}}{4+|6 l+3|} & \text { при } & n=3,
\end{array}
$$

где $\omega=|e B| / m c-$ циклотронная частота.

Следует отметить, что решениям в виде полиномов в квантовой механике отвечают в классической механике решения, соответствующие существованию замкнутых траекторий частиш в рассматриваемых полях при выполнении соотношений типа (52).

\section{6. МЕТОД ФАКТОРИЗАЦИИ}

Точные решения изучаемых уравнений в случае суперпозиции внешних сильных кулоновского и магнитного полей можно найти в виде произведения некоторой весовой функции и конечного полинома. Для этого используется метод, близкий методу факторизации нерелятивистской квантовой механики (подробно о методе факторизации см. в книге [18]). Этот метод позволяет находить спектр любого эрмитова оператора, собственные значения которого ограничены снизу. Для этого оператор, спектр которого требуется найти, представляется в виде произведения операторов типа операторов рождения и уничтожения $a^{+}, a$, используемых для приведения гамильтониана гармонического осциллятора к виду $H_{\mathrm{osc}}=\hbar \omega\left(a^{+} a+1 / 2\right)$.

Решения уравнения (25) будем искать в виде

$$
F(r)=r^{\gamma} \exp \left(-\frac{a r^{2}}{2}\right) f(r)
$$

где $\gamma$ и $а$ те же, что и в предыдушем разделе. Подставляя формулу (53) в уравнение (25), получим

$$
\left[\frac{d^{2}}{d x^{2}}+\left(\frac{2 \gamma}{x}-2 x\right) \frac{d}{d x}+\left(K+\frac{b}{x}\right)\right] f(x)=0
$$


где

$$
K=\frac{\left[E^{2}-m^{2}-2 a(l+2+\gamma)\right]}{a}, \quad b=\frac{2 E Z \alpha}{\sqrt{a}} .
$$

Видно, что проблема нахождения спектра для уравнения (25) оказывается эквивалентной проблеме определения спектра собственных значений оператора [6]

$$
H=-\frac{d^{2}}{d x^{2}}-\left(\frac{2 \gamma}{x}-2 x\right) \frac{d}{d x}-\frac{b}{x} .
$$

Оператор (56) ограничен и может быть представлен в виде

$$
H=a^{+} a+p,
$$

где $p$ имеет смысл квантового числа данного оператора, причем $p=K$. Собственные функции оператора $H$ при $p=0$ должны удовлетворять уравнению

$$
a \psi=0 .
$$

Если решения в виде полиномов существуют для уравнения (58), то оператор $a$, очевидно, должен иметь вид

$$
a=\frac{\partial}{\partial x}-\sum_{k=1}^{s} \frac{1}{x+x_{k}},
$$

где $x_{k}$ - корни, а $s$ - степень соответствуюшего полинома. Тогда оператор $a^{+}$будет иметь вид

$$
a^{+}=-\frac{\partial}{\partial x}-\frac{2 \gamma}{x}+2 x-\sum_{k=1}^{s} \frac{1}{x+x_{k}} .
$$

Подставляя выражения (59) и (60) в уравнение (57) и затем сравнивая (56) и (57), получим систему уравнений для определения корней $x_{k}$

$$
\frac{2 \gamma}{x_{k}}-2 x_{k}-2 \sum_{j \neq k}^{s} \frac{1}{x_{j}-x_{k}}=0
$$

и соотношение

$$
-b=2 \gamma \sum_{k=1}^{s} x_{k}^{-1}, \quad \text { где } \quad p=s .
$$

Суммируя уравнения системы (61), последнее соотношение можно привести к виду

$$
-b=2 \sum_{k=1}^{s} x_{k}, \quad \text { где } \quad p=s .
$$

Из этих формул мы снова можем найти простейшие решения (для $s=1,2$ ), а также значения энергии и напряженности магнитного поля, которые совпадают с решениями (47)-(50). 
Заметим, что в нерелятивистском случае проблему можно свести к нахождению решений и спектра собственных значений энергии следующего уравнения Шредингера (для функции $f(r)$ ), которое нетрудно получить из $(24)$ в виде

$$
\left(\frac{d^{2}}{d x^{2}}+\frac{1}{x} \frac{d}{d x}-\frac{x^{2}}{4}-\frac{l^{2}}{4 x^{2}} \pm \frac{b}{x}+\beta\right) f(x)=0 .
$$

Здесь $x=r / l_{B}, \beta=2 E / \hbar \omega-(l+1)$,

$$
b=\frac{l_{B}}{a_{\mathrm{B}}} \equiv Z \alpha \sqrt{\frac{2 m c^{2}}{\hbar \omega}} .
$$

В нерелятивистском случае мы рассмотрим кулоновское взаимодействие, соответствующее как притяжению, так и отталкиванию, имея в виду, что двумерная система двух электронов в однородном магнитном поле (перпендикулярном плоскости движения электронов) представляет большой интерес для описания квантового эффекта Холла. Верхний (нижний) знак в уравнении (64) соответствует отталкиванию (притяжению). При $b=0$, когда кулоновским взаимодействием можно пренебречь, решения представляются через обобшенные полиномы Лагерра, а спектр энергий $E_{n}-$ уровнями Ландау

$$
E_{n}=\hbar \omega\left(n_{r}+\frac{l+|l|+2}{2}\right), \quad n_{r}=0,1,2, \ldots .
$$

Чтобы построить точные решения уравнения (64), в полученных выше общих соотношениях, очевидно, достаточно положить $2 \gamma=2|l|+1, b=2 m Z \alpha / \sqrt{a}$, а также учесть, что $p=E / \hbar \omega-(|l|+l+2)$. Искомый полином имеет вид (с точностью до нормировки)

$$
\prod_{k=1}^{s}\left(x+x_{k}\right)=x^{s} \mp b x^{s-1}+\cdots+(-1)^{s}(2|l|+1)
$$

где учтено, что

$$
\mp b=\sum_{k=1}^{s} x_{k}
$$

и

$$
\prod_{k=1}^{s} x_{k}=2|l|+1
$$

Для $s=1,2$ корни полинома $x_{k}$ и значения параметра $b$, при которых решения в виде полиномов соответствующих степеней сушествуют, нетрудно найти из формул (61) и (68), соответственно, в виде

$$
\begin{gathered}
x_{1}=\mp(2|l|+1), \quad b=\sqrt{2|l|+1} \quad \text { при } \quad s=1, \\
x_{1}=\frac{2|l|+1}{x_{2}}, \quad x_{2}=\mp \frac{1 \pm \sqrt{4|l|+3}}{\sqrt{2}}, \quad b=\sqrt{2(4|l|+3)} \quad \text { при } \quad s=2 .
\end{gathered}
$$


Из соотношений (65) и (69) следуют значения напряженности магнитного поля, при которых искомые решения сушествуют,

$$
\begin{array}{lll}
\hbar \omega=2 m c^{2} \frac{(Z \alpha)^{2}}{2|l|+1} & \text { при } & s=1, \\
\hbar \omega=m c^{2} \frac{(Z \alpha)^{2}}{4|l|+3} & \text { при } & s=2,
\end{array}
$$

а также соответствуюшие значения энергии

$$
\begin{aligned}
& E_{1}=\frac{2 m\left(Z e^{2}\right)^{2}}{\hbar^{2}(2|l|+1)}(3+l+|l|), \\
& E_{2}=\frac{m\left(Z e^{2}\right)^{2}}{\hbar^{2}(4|l|+3)}(4+l+|l|) .
\end{aligned}
$$

Формулы (70) и (71) согласуются с результатами работы [7]. Они незначительно отличаются от формул (52) и (51), т.к. последние были получены с помощью уравнения (25) (в нерелятивистском приближении), которое справедливо лишь при малых $r$.

Полиномы с $s=1,2$ имеют вид

$$
x+x_{1}=x \pm b, \quad \prod_{k=1}^{2}\left(x+x_{k}\right)=x^{2} \pm b x+2|l|+1
$$

где верхние (нижние) знаки соответствуют случаю кулоновского отталкивания (притяжения). Волновая функция электрона определяется формулой (53). Для $s=1,2$ в случае кулоновского отталкивания волновые функции не имеют узлов, т.е. обе функции описывают основные состояния, в то время как в случае кулоновского притяжения волновые функции для $s=1,2$ имеют один и два узла (функции описывают возбужденные состояния).

Для $s=1,2$ найденные решения (70), (71) и (72) единственны, т.к. только для $s=1,2$ параметр $b$ и корни полиномов, а значит, циклотронная частота и полином (67), определяются вполне однозначно уравнениями (61) и (62). Для $s \geqslant 3$ уравнения (61) и (62) имеют более одного решения, а именно, число решений равно $\operatorname{int}(s+1) / 2$. Поэтому для $s \geqslant 3$ даже в случае кулоновского отталкивания решения будут соответствовать как основным состояниям (это решение с наименьшей частотой и не имеюшее узлов), так и возбужденным состояниям.

Отметим, что метод факторизации подобен рассмотрению задачи для $b \neq 0$ с помошью суперсимметричной нерелятивистской квантово-механической модели Виттена [19] для метрики [6, 20]

$$
L^{2}\left(0, \infty ; \exp \left(-\frac{x^{2}}{2}\right) x^{2|l|+1} d x\right) .
$$

Подчеркнем, что метод факторизации приводит к тем же результатам, что и метод рекуррентных соотношений. 


\section{Список литературы}

[1] The Quantum Hall Effect. $2^{\text {nd }}$ ed. Eds. R.E. Prange, S.M. Girvin. New York: Springer-Verlag, 1990.

[2] F. Wilczek. Fractional Statistics and Anyon Superconductivity. Singapore: World Scientific, 1990.

[3] A.M. J. Schakel. Phys. Rev. D. 1991. V. 43. P. 1428; A. Neagu, A. M. J. Schakel. Phys. Rev. D. 1993. V. 48. P. 1785.

[4] A. M. J. Schakel, G. W. Semenoff. Phys. Rev. Lett. 1991. V. 66. P. 2653.

[5] В. Г. Багров, Д. М. Гитман, И. М. Тернов, В. Р. Халилов. Точные решения релятивистских волновых уравнений. Новосибирск: Наука, 1984.

[6] B. A. Lysov, O.F. Dorofeyev, I. M. Ternov. Nucl. Instr. Methods. A. 1991. V. 308. P. 115.

[7] M. Taut. J. Phys. Math. Gen. A. 1995. V. 28. P. 2081.

[8] V. R. Khalilov, C. L. Ho. Mod. Phys. Lett. A. 1998. V. 13. P. 615.

[9] I. M. Ternov, V. V. Mikhailin, V.R. Khalilov. Synchrotron Radiation and Its Applications. Amsterdam: Harwood Academic Pub., 1985.

[10] В. Б. Берестецкий, Е. М. Лифиич, Л. П. Питаевский. Квантовая электродинамика. М.: Наука, 1980.

[11] А.А. Соколов, И. М. Тернов. Релятивистский электрон. М.: Наука, 1974.

[12] Я. Б. Зельдович, В. С. Попов. УФН. 1971. Т. 105. С. 403.

[13] А. Б. Мигдал. Фермионы и бозоны в сильных полях. М.: Наука, 1978.

[14] А. А. Гриб, С. Г. Мамаев, В. М. Мостепаненко. Вакуумные квантовые эффекты в сильных полях. М.: Энергоатомиздат, 1988.

[15] I. Pomeranchuk, Ya. Smorodinsky. J. Phys. USSR. 1945. V. 9. P. 97.

[16] V. M. Villalba, R. Pino. Analytic Solution of a Relativistic Two-dimensional Hydrogen-like Atom in a Constant Magnetic Field. cond-mat/9712044.

[17] Ф. Олвер. Введение в асимптотические методы и специальные функции. М.: Наука, 1978.

[18] П. В. Елютин, В. Д. Кривченков. Квантовая механика. М.: Наука, 1976.

[19] E. Witten. Nucl. Phys. B. 1981. V. 188. P. 513.

[20] М. Рид, Б. Саймон. Методы современной математической физики. Т. 2. Гармонический анализ. Самосопряженность. М.: Мир, 1978. 